\title{
CONVOLUTIONS OF SCHLICHT FUNCTIONS
}

\author{
M. S. ROBERTSON
}

1. Introduction. Let $f(z)$ and $g(z)$ be analytic functions regular and schlicht for $|z|<1$ and defined by the power series

$$
\begin{aligned}
& f(z)=z+a_{2} z^{2}+\cdots+a_{n} z^{n}+\cdots, \\
& g(z)=z+b_{2}+\cdots+b_{n} z^{n}+\cdots .
\end{aligned}
$$

Let $F(z)$, the convolution of $f(z)$ and $g(z)$, be given by

$$
F(z)=z+a_{2} b_{2} z^{2}+\cdots+a_{n} b_{n} z^{n}+\cdots,
$$

and let $G(z)$ be the related function defined by

$$
G(z)=\int_{0}^{z} \frac{F(z)}{z} d z=z+\sum_{2}^{\infty} \frac{a_{n} b_{n}}{n} z^{n} .
$$

A little over a decade ago the author [6] showed that if $f(z)$ and $g(z)$ are real on the real axis and univalently convex in $|z|<1$ then $F(z)$ is schlicht and convex in the direction of the imaginary axis for $|z|<1$. In a recent paper [5] Pólya and Schoenberg have conjectured that if $f(z)$ and $g(z)$ are convex in $|z|<1$ then so is $F(z)$. These authors attribute to Mandelbrojt and Schiffer another conjecture that $G(z)$ is schlicht in $|z|<1$ whenever $f(z)$ and $g(z)$ are schlicht in $|z|<1$. Soon afterwards Epstein and Schoenberg [3], and Loewner and Netanyahu [4], showed that this last conjecture was false and it is possible for $G^{\prime}(z)$ to have a zero within the unit circle.

It is natural to enquire what can be said in the meromorphic case when $f(z)$ and $g(z)$ each have a simple pole at the origin. In view of the negative nature of the results mentioned above it is somewhat surprising that the following theorem, very easily proved, seems to have escaped notice before this time.

THEOREM 1. Let

$$
\begin{aligned}
& f(z)=\frac{1}{z}+\sum_{1}^{\infty} a_{n} z^{n}, \\
& g(z)=\frac{1}{z}+\sum_{1}^{\infty} b_{n} z^{n}
\end{aligned}
$$

Presented to the Society, September 14, 1961; received by the editors April 21, 1961. 
be regular and schlicht for $0<|z|<1$. Then

$$
F(z)=\frac{1}{z}+\sum_{1}^{\infty} a_{n} b_{n} z^{n}
$$

is also regular and schlicht for $0<|z|<1$. Furthermore, $F(z)$ is closeto-convex for $0<|z|<1$, is continuous on $|z|=1$, and satisfies

$$
\operatorname{Re}\left\{z^{2} F^{\prime}(z)\right\}<0, \quad|z|<1
$$

$$
\int_{\theta_{1}}^{\theta_{2}} \operatorname{Re}\left\{1+\frac{z F^{\prime \prime}(z)}{F^{\prime}(z)}\right\} d \theta<\pi-\left(\theta_{2}-\theta_{1}\right)
$$

for $0<\theta_{2}-\theta_{1} \leqq 2 \pi, z=r e^{i \theta}, 0<r<1$,

$$
\left|F\left(e^{i \theta_{2}}\right)-F\left(e^{i \theta_{1}}\right)\right| \leqq 2\left|e^{i \theta_{2}}-e^{i \theta_{1}}\right| \text {. }
$$

CoROLlary. The conclusions of Theorem 1 pertaining to $F(z)$ apply also to all the partial sums $F_{n}(z)$ of (1.7),

$$
F_{n}(z)=\frac{1}{z}+\sum_{k=1}^{n} a_{k} b_{k} z^{k}
$$

2. Proof of Theorem 1. We prove first that $F(z)$, defined as in (1.7) is schlicht in $0<|z|<1$. If $z_{1}$ and $z_{2}$ are two distinct points of the annulus $0<|z|<1$, we have

$$
\begin{aligned}
& \frac{F\left(z_{2}\right)-F\left(z_{1}\right)}{z_{2}-z_{1}}=\frac{-1}{z_{1} z_{2}}+\sum_{1}^{\infty} a_{n} b_{n}\left(z_{2}^{n-1}+z_{2}^{n-2} z_{1}+\cdots+z_{1}^{n-1}\right) \\
& \left|\frac{F\left(z_{2}\right)-F\left(z_{1}\right)}{z_{2}-z_{1}}\right|>1-\sum_{1}^{\infty} n\left|a_{n} b_{n}\right| \\
& \geqq 1-\left[\sum_{1}^{\infty} n\left|a_{n}\right|^{2} \cdot \sum_{1}^{\infty} n\left|b_{n}\right|^{2}\right]^{1 / 2} \\
& \geqq 0 \text {, }
\end{aligned}
$$

since by the area theorem for schlicht functions

$$
\sum_{1}^{\infty} n\left|a_{n}\right|^{2} \leqq 1, \quad \sum_{1}^{\infty} n\left|b_{n}\right|^{2} \leqq 1 .
$$

This proves that $F(z)$ is schlicht in $0<|z|<1$.

$F(z)$ is also continuous on $|z|=1$ and satisfies the Lipschitz condition (1.10). If $\left|z_{1}\right|=\left|z_{2}\right|=1$ we have from (2.1) 


$$
\begin{aligned}
\left|\frac{F\left(z_{2}\right)-F\left(z_{1}\right)}{z_{2}-z_{1}}\right| & \leqq 1+\sum_{1}^{\infty} n\left|a_{n} b_{n}\right| \\
& \leqq 1+\left[\sum_{1}^{\infty} n\left|a_{n}\right|^{2} \cdot \sum_{1}^{\infty} n\left|b_{n}\right|^{2}\right]^{1 / 2} \leqq 2 .
\end{aligned}
$$

We next observe that

(2.5) $\operatorname{Re} z^{2} F^{\prime}(z)=-1+\operatorname{Re} \sum_{1}^{\infty} n a_{n} b_{n} z^{n+1}<-1+\sum_{1}^{\infty} n\left|a_{n} b_{n}\right| \leqq 0$.

We shall say that a function $\psi(z)$

$$
\psi(z)=\frac{1}{z}+\sum_{0}^{\infty} c_{n} z^{n}
$$

regular in $0<|z|<1$, is close-to-convex in $0<|z|<1$ if there exists a star-like schlicht function $\phi(z)$

$$
\phi(z)=\frac{d_{-1}}{z}+\sum_{0}^{\infty} d_{n} z^{n}, \quad 0<|z|<1,
$$

such that

$$
\operatorname{Re} \frac{z \psi^{\prime}(z)}{\phi(z)}>0, \quad|z|<1 .
$$

From (2.5) it follows that $F(z)$ is close-to-convex with respect to the function $\phi(z)=-z^{-1}$.

Let $P(r, \theta), Q(\theta)$ be defined by the equations

$$
\begin{aligned}
& P(r, \theta)=\arg z F^{\prime}(z)=\arg F^{\prime}(z)+\theta, \quad z=r e^{i \theta}, \\
& Q(\theta)=\arg (-1 / z)=\pi-\theta, \quad 0 \leqq \theta<2 \pi .
\end{aligned}
$$

Since from (2.5) we have $\operatorname{Re} z F^{\prime}(z) /(-1 / z)>0,|z|<1$, there exists an integer $k$ so that

$$
|P(r, \theta)-Q(\theta)+2 k \pi|<\pi / 2 \text {. }
$$

By an appropriate choice of the definition of the argument of $z F^{\prime}(z)$ we may take $k=0$. For $0<\theta_{2}-\theta_{1} \leqq 2 \pi$

$$
\begin{aligned}
P\left(r, \theta_{2}\right)-P\left(r, \theta_{1}\right)= & \left\{P\left(r, \theta_{2}\right)-Q\left(\theta_{2}\right)\right\} \\
& +\left\{Q\left(\theta_{1}\right)-P\left(r, \theta_{1}\right)\right\}+\left\{Q\left(\theta_{2}\right)-Q\left(\theta_{1}\right)\right\} \\
< & \pi / 2+\pi / 2+\left\{\left(\pi-\theta_{2}\right)-\left(\pi-\theta_{1}\right)\right\} \\
= & \pi \quad\left(\theta_{2}-\theta_{1}\right) .
\end{aligned}
$$


Since the inclination of the tangent vector to the image curve of $|z|=r$ by the mapping $w=F(z)$ is $P(r, \theta)+\pi / 2$ we may interpret (2.12) as meaning that as the tangent line rotates with $\theta$ advancing from $\theta_{1}$ to $\theta_{2}$ the tangent does not turn back on itself through an angle as large as $\pi-\left(\theta_{2}-\theta_{1}\right)$. Since for $z=r e^{i \theta}$ we have

$$
\int_{\theta_{1}}^{\theta_{2}} \operatorname{Re}\left[1+\frac{z F^{\prime \prime}(z)}{F^{\prime}(z)}\right] d \theta=P\left(r, \theta_{2}\right)-P\left(r, \theta_{1}\right)
$$

the inequality (2.12) is equivalent to (1.9).

For $\alpha \geqq 0$ let us now define an analytic function $F_{\alpha}(z)$ by the equation

$$
F_{\alpha}(z)=\frac{1}{z}+\sum_{1}^{\infty} n^{\alpha} a_{n} b_{n} z^{n}, \quad|z|<1,
$$

where $a_{n}, b_{n}$ are the coefficients defined by (1.5) and (1.6). Is $F_{\alpha}(z)$ schlicht for some positive $\alpha$ for every schlicht $f(z)$ and $g(z)$ in $0<|z|$ $<1$ ? We have seen that $F_{0}(z)$ is always schlicht. We shall now show that $F_{1}(z)$ need not be schlicht in $0<|z|<1$. Clunie [1] recently has given an example of a schlicht function $f(z), 0<|z|<1$,

$$
f(z)=\frac{1}{z}+\sum_{1}^{\infty} a_{n} z^{n}
$$

for which $n a_{n}$ is not bounded. Taking $g(z)$ in (1.6) to be $z^{-1}+n^{-1} z^{n}$, then $F_{\alpha}(z)$ in $(2.12)$ becomes

$$
F_{\alpha}(z)=\frac{1}{z}+n^{\alpha}\left(\frac{1}{n}\right) a_{n} z^{n}
$$

If $F_{\alpha}(z)$ is schlicht in $0<|z|<1$, we must have $F_{\alpha}^{\prime}(z) \neq 0$ which requires that

$$
n^{\alpha}\left(\frac{1}{n}\right)\left|a_{n}\right| \leqq \frac{1}{n} .
$$

If $\alpha=1,(2.17)$ implies that $n a_{n}$ is bounded which is not the case. In this case $F_{1}(z)$ is not schlicht in $0<|z|<1$.

If in (1.1) we have $\sum_{2}^{\infty} n\left|a_{n}\right| \leqq 1$ it is well-known [2] that $f(z)$ is starlike in $|z|<1$. We have seen that $F(z)$ of (1.7) satisfies the analogous inequality

$$
\sum_{1}^{\infty} n\left|a_{n} b_{n}\right| \leqq 1
$$


and $F(z)$ is close-to-convex and schlicht. Does (2.18) imply that $F(z)$ is also starlike in $0<|z|<1$ ?

Remark (added in proof): I am indebted to Professor Christian Pommerenke for informing me that (2.18) implies that $F(z)$ is starlike in $0<|z|<1$. The following proof, somewhat different from his, easily establishes this fact.

$$
\begin{aligned}
& \left|z F^{\prime}(z)+F(z)\right|-\left|z F^{\prime}(z)-F(z)\right| \\
& \quad=\left|\sum_{1}^{\infty}(n+1) a_{n} b_{n} z^{n}\right|-\left|-\frac{2}{z}+\sum_{1}^{\infty}(n-1) a_{n} b_{n} z^{n}\right| \\
& \quad \leqq \sum_{1}^{\infty}(n+1)\left|a_{n} b_{n}\right| r^{n}-\left[\frac{2}{r}-\sum_{1}^{\infty}(n-1)\left|a_{n} b_{n}\right| r^{n}\right] \\
& \quad<\frac{2}{r}\left(\sum_{1}^{\infty} n\left|a_{n} b_{n}\right|-1\right)<0 .
\end{aligned}
$$

It follows from (2.19) that if $P(z)=-z F^{\prime}(z) / F(z)$ then

$$
\left|\frac{P(z)-1}{P(z)+1}\right|<1, \quad \operatorname{Re} P(z)>0,
$$

and consequently $F(z)$ is starlike in $0<|z|<1$.

\section{REFERENCES}

1. J. Clunie, On schlicht functions, Ann. of Math. (2) 69 (1959), 511-519.

2. - On meromorphic schlicht functions, J. London Math. Soc. 34 (1959), 215-216.

3. B. Epstein and I. J. Schoenberg, On a conjecture concerning schlicht functions, Bull. Amer. Math. Soc. 65 (1959), 273-275.

4. C. Loewner and E. Netanyahu, On some compositions of Hadamard type in classes of analytic functions, Bull. Amer. Math. Soc. 65 (1959), 284-286.

5. G. P6lya and I. J. Schoenberg, Remarks on de la Vallee Poussin means and convex conformal maps of the circle, Pacific J. Math. 8 (1958), 295-334.

6. M. S. Robertson, Applications of a lemma of Fejer to typically-real functions, Proc. Amer. Math. Soc. 1 (1950), 555-561.

Rutgers, The State University 